\title{
Research on Analysis of Eddy Current Response to Lay Length of Wire Ropes
}

\author{
Shaoni Jiao ${ }^{\mathrm{a}, \mathrm{b}, \mathrm{c}}$, Xiaojuan Jia ${ }^{\mathrm{d}, 1}$, Tengyu $\mathrm{Li}^{\mathrm{a}}{ }^{\mathrm{a}, \mathrm{c}}$, Shukui Jin ${ }^{\mathrm{a}, \mathrm{b}, \mathrm{c}}$, Ziming Kou ${ }^{\mathrm{a}, \mathrm{b}, \mathrm{c}}$, Juan \\ $\mathrm{Wu}^{\mathrm{a}, \mathrm{b}, \mathrm{c}}$, Yanfei Kou ${ }^{\mathrm{e}}$ \\ ${ }^{a}$ College of Mechanical and Vehicle Engineering, Taiyuan University of Technology, \\ Taiyuan,030024 \\ ${ }^{\mathrm{b}}$ Shanxi Province Engineering Technology Research Center for Mine Fluid Control, \\ Taiyuan, 030024 \\ ${ }^{\mathrm{c}}$ National-local Joint Engineering Laboratory of Mining Fluid Control, Taiyuan, \\ 030024 \\ ${ }^{\mathrm{d}}$ College of Mechanical and Equipment Engineering, Hebei University of Engineering, \\ Handan, 056038 \\ ${ }^{\mathrm{e}}$ College of Mechanical Engineering, North Central University, Taiyuan, 030051
}

\begin{abstract}
The lay length is one of the important parameters for the wire rope and its detection can indirectly characterize its stress and surface damage state. The eddy current testing $(E C T)$ method is advantageous because of its easy operation, single-side detection, low requirement of surface preparation, applicability in harsh environment, and other properties. Hence the lay length of the wire ropes is measured by ECT in this paper. The eddy current (EC) response to the wire rope is simulated. The EC on the surface of the strand below the pancake coil forms a vortex, and the distribution of the EC on the curved surfaces on both sides of the strand is symmetrical. The spacing between the adjacent peaks or troughs of the scanning signal is the distance between the adjacent strands, and the number of peaks or troughs of the scanning signal in a range of the lay length is related to the number of strands of the wire rope. Finally, the experimental validation is performed. The relative error of the lay length of the wire rope assessed by the EC method is less than $0.28 \%$.
\end{abstract}

Keywords. Wire rope, Lay length, Eddy current testing, Finite element analysis

\section{Introduction}

The wire rope is a flexible space spiral structure winded by the same size of the strands, and has been widely utilized as a bearing and transmission component in the fields of mining, tourism, construction, et al[1]. Guaranteeing the safety of the wire rope is important for reducing economic loss or casualties. The tension or deformation of the wire rope caused by surface wear can lead to changes in the lay length of the wire rope [2]. Therefore, the detection of the lay length can indirectly characterize the force and surface damage of the wire rope. The traditional method of measuring the lay length is mainly done manually, which is not suitable for detecting the running wire rope.

\footnotetext{
${ }^{1}$ Corresponding author: Xiaojuan Jia. Email: jiaxiaojuan@cqu.edu.cn.
} 
Different nondestructive methods have been applied to detect the running wire ropes, such as the image recognition method and the magnetic flux leakage (MFL) method. Alberto has proposed an automatic vision measurement system for the lay length detection. The image of the running wire rope is acquired through the camera, and then the wireline contour is extracted using the image and signal processing algorithms to determine the lay length. The accuracy of the lay length obtained by this method is $1.2 \mathrm{~mm}[3]$. Esther has established an image comprehensive analysis system based on the wire rope model, which can capture the actual 2-D projection of the wire rope using the camera, and construct a parametric 3-D model based on the structural parameters of the wire rope model. The accuracy of the lay length and strand space obtained by this method is $1 \mathrm{~mm}$ [4]. Sylvain has calculated the lay length according to the change of the scanning MFL signal with the uneven surface of the wire rope [5].

The eddy current testing (ECT) method has a series of advantages and has been applied in the wire rope testing. Cao has quantitatively tested the broken wires with symmetrically arranged the excitation and the detection coils around the wire rope [6]. Zheng has adopted a double eddy current (EC) probe placed in parallel to detect the lay length. The sensitivity is related to the distance between the probe and the probe consistency. Hence the detection difficulty is increased [7].

The image recognition method is greatly affected by the external environment. The MFL method requires the wire rope to be magnetized and its operation is relatively complicated. In this paper, the ECT for detecting the lay length of the wire rope is studied. Firstly, the EC distribution on the surface of a single strand is analyzed and the scanning signal of the wire rope with the differential bridge is obtained. Then the experimental platform is established for lay length detection.

\section{Finite element analysis}

\section{1. $\boldsymbol{A}_{r}, V-\boldsymbol{A}_{r}$ formulation}

The governing equations for the calculation of EC response to the wire rope are as follows

$$
\begin{aligned}
& -\frac{1}{\mu_{0}} \nabla^{2} \boldsymbol{A}_{r}+j \omega \sigma\left(\boldsymbol{A}_{r}+\nabla v\right)=-\nabla \times \boldsymbol{H}_{s}-j \omega \sigma \boldsymbol{A}_{s} \quad\left(\Omega_{1}\right) \\
& \nabla \cdot\left(-j \omega \sigma \boldsymbol{A}_{r}-j \omega \sigma \nabla v\right)=\nabla \cdot j \omega \sigma \boldsymbol{A}_{s} \quad\left(\Omega_{1}\right) \\
& -\frac{1}{\mu_{0}} \nabla^{2} \boldsymbol{A}_{r}=0 \quad\left(\Omega_{2}\right)
\end{aligned}
$$

where the wire rope model area $\left(\Omega_{1}\right)$ and the air domain containing the power supply $\left(\Omega_{2}\right)$ constitute the entire solution domain. In (1)-(3), $\boldsymbol{A}_{r}$ stands for the simplified magnetic vector potential (MVP). $\boldsymbol{A}_{s}$ and $\boldsymbol{H}_{s}$ represent the magnetic vector position and magnetic field strength generated by the excitation current in the solution space, respectively. $\mu_{0}$ represents the magnetic permeability of the solution space. $\omega$ stands for the angular frequency. In (1) and (2), $v=V /(j \omega)$, in which $V$ represents the simplified electrical scalar position. The eddy current density $\boldsymbol{J}$ can be calculated as follows 


$$
\boldsymbol{J}=-j \omega \sigma(\boldsymbol{A}+\nabla v)
$$

where $\boldsymbol{A}$ is the vector sum of $\boldsymbol{A}_{s}$ and $\boldsymbol{A}_{r}$.

\subsection{Simulation model}

The model includes the wire rope and the probe composed of a double pancake coil, as shown in Fig. 1. The Oxyz global coordinate system is established and the origin is the wire rope center. Figs.1(a) and (b) are the projections of the model on the $x z$ and $y z$ planes, respectively. The wire rope has the total length of $240 \mathrm{~mm}$, the lay length of 60 $\mathrm{mm}$, the radius of the single helix is $3.48 \mathrm{~mm}$, the strand radius of $1.65 \mathrm{~mm}$. The conductivity is $3.0 \times 10^{7} \mathrm{~S} / \mathrm{m}$ and the relative magnetic permeability of the wire rope is 10[8]. The coils are coaxially placed and are the same size. Each pancake coil has the inner diameter of $6.0 \mathrm{~mm}$, the outer diameter of $8.0 \mathrm{~mm}$, the height of $3.0 \mathrm{~mm}$, the number of turns of 500 , the lifting height of $0.5 \mathrm{~mm}$ and the distance of $2.5 \mathrm{~mm}$ between the upper and lower coils. The exciting frequency is $80 \mathrm{kHz}$. The model is solved by an FE analysis software package developed in lab.

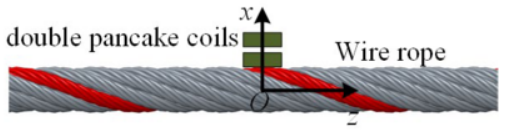

Fig.1. Schematic of the simulation model (a) the $x z$ plane view (b) the $y z$ plane view

\subsection{Eddy current distribution of single strand}

Fig. 2 shows the partial single strand and the probe which remains the same with the one in Section 2.2. The surface of the strand is divided into four parts. The bottom surface of the probe is just on Surface 1. Surface 3 is the corresponding back side of Surface 1 . Surfaces 2 and 4 are the corresponding side faces of the strand.
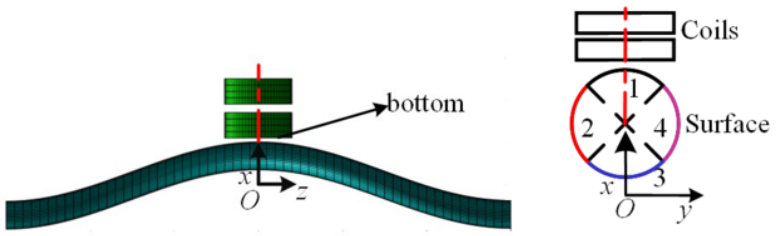

Fig.2. The single strand model with the probe

Fig. 3 shows the distribution of the real part of the EC density at the bottom center of the probe $(x=5.63 \mathrm{~mm}, y=0, z=0)$. Fig. 3 (a) is the partial distribution of the EC density on Surface 1. A vortex is formed on Surface 1 and the center of the vortex denoted by point $A$ is located just under the bottom center of the probe. Fig. 3 (b)-(d) are the partial distributions of the EC density on Surface 2-4. ECs are formed on Surface 2-4 and the centers are denoted by point $B$, point $C$ and point $D$. The distribution of ECs in the wire rope is different from the one in the traditional flat metal due to the special helical structure. When the probe scans along the axis of the wire rope, the response of EC will change with the distribution of ECs. Hence the lay length will be deduced from the change of the response of EC. 


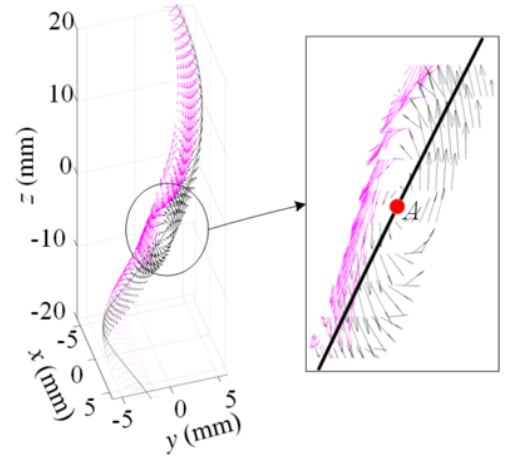

(a)

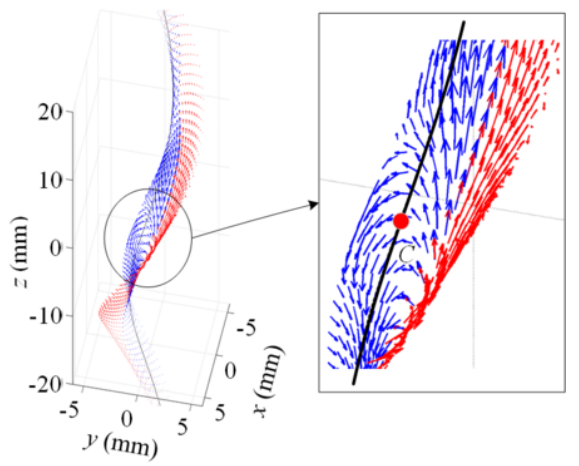

(c)

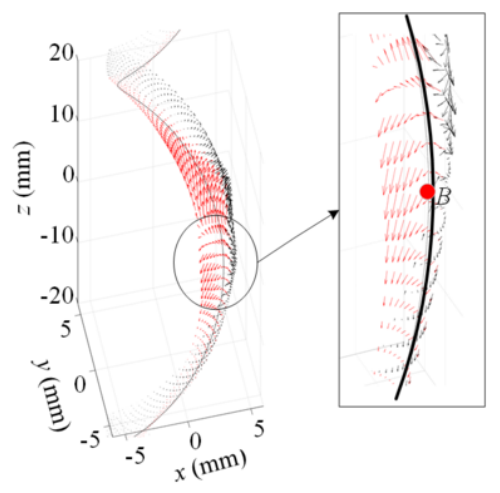

(b)

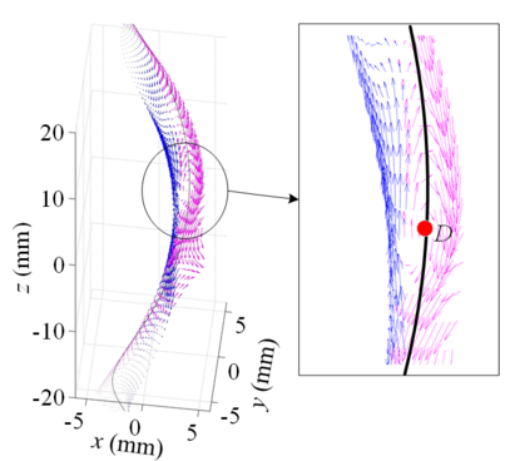

(d)

Fig.3.The real part distribution of the EC density on the single strand surfaces (a) on Surface 1(b) on Surface 2(a) on Surface 3(a) on Surface 4

\subsection{Linear scan simulation}

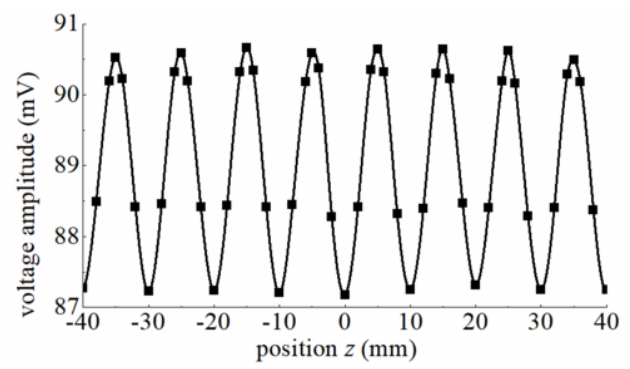

Fig.4. Simulation result of scanning the wire rope

The probe scans from $z=-40 \mathrm{~mm}$ to $z=40 \mathrm{~mm}$ with a step of $2 \mathrm{~mm}$. Fig. 4 shows the change of the amplitude of the differential voltage with the scanning position. It can be seen that the curve changes periodically and there are 7 peaks or troughs in one lay length range. The spacing between the adjacent peaks or troughs is just the gap of the wire strand. 


\section{Experiments}

\subsection{Eddy current testing system}

To validate the simulation result shown in Fig.4, the experiment of detecting the lay length by the EC method is conducted. Fig.5 shows the platform and is mainly composed of the signal generator (DG4102), the probe, the electronically controlled displacement platform, the Native Instruments (NI) data acquisition card and the monitor. The excitation signal is with the frequency of $80 \mathrm{kHz}$ and the amplitude of 10 $\mathrm{V}$. The acquired data is post-processed by Labview.

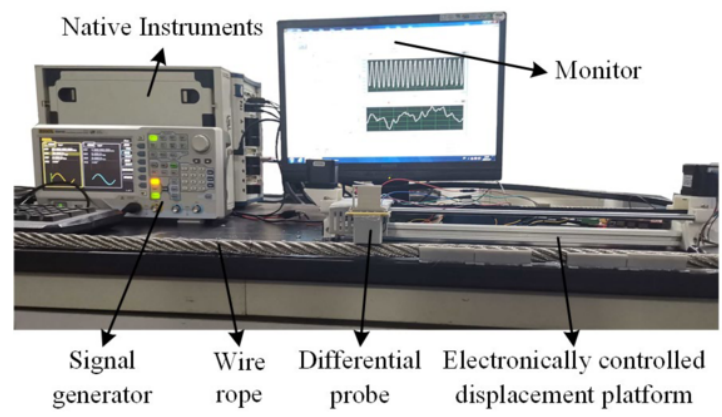

Fig. 5 The experimental platform of detecting the lay length

Fig. 6 shows the $6 \times 19+$ IWRC wire rope sample with a diameter of $16.0 \mathrm{~mm}$ and a total length of $1200 \mathrm{~mm}$. The average lay length and gap between the two adjacent strands are $104 \mathrm{~mm}$ and $17.33 \mathrm{~mm}$, respectively. Fig 7 shows the probe with two same pancake coils arranged coaxially. The probe is connected to the differential detection circuit printed on the PCB board.

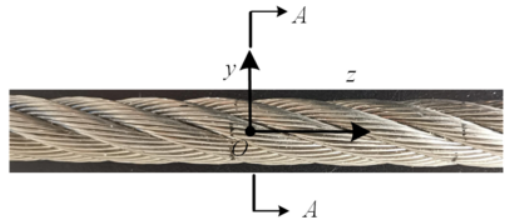

Fig.6. $6 \times 16+$ IWS wire rope sample

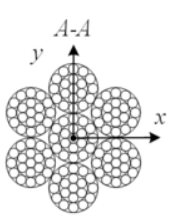

Fig.7. The probe for the lay length detection

\subsection{Linear scanning test}

Fig. 8 shows the results of scanning the wire rope with a total scan length of $360 \mathrm{~mm}$. The experiment is repeated twice and the change of the amplitude of the differential voltage with the scanning position is shown in Fig.8. The results agree well during the two experiments. The amplitude of the differential voltage exhibits alternate peaks and valleys during the scanning. The distance between the two adjacent peaks or valleys is a gap between the adjacent strands of the wire rope. The distance between the adjacent seven peaks or valleys is approximately one lay length, which is consistent with the simulation results shown in Fig.4. The average strand gap and lay length are $17.16 \mathrm{~mm}$ and $103.71 \mathrm{~mm}$ respectively. The relative error is the difference between the manually 
measured lay length and the value assessed by the EC method divided by the manually measured value and is $0.28 \%$ in Fig. 8 .

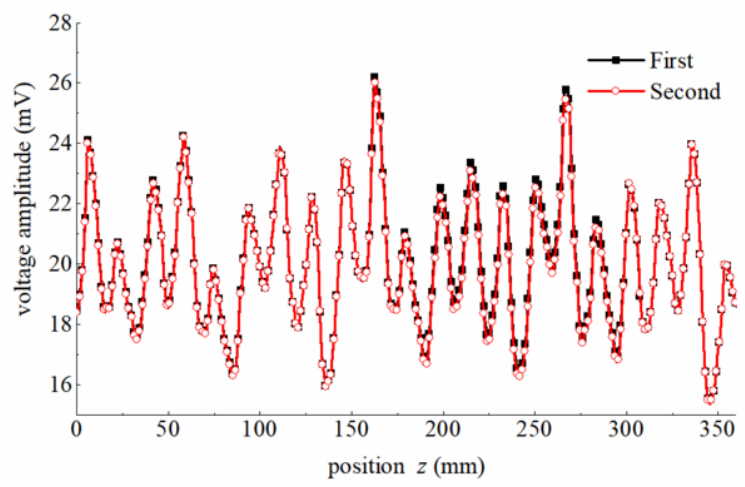

Fig.8. Experimental result of scanning the wire rope

\section{Conclusions}

The lay length of the wire rope is detected by the EC method in this paper. The $\boldsymbol{A}_{\mathrm{r}}, V-\boldsymbol{A}_{\mathrm{r}}$ formulation for calculating the EC response to the wire rope is established. The finite element analysis shows that the EC forms vortex on the surface of the single helical strand. Due to the uneven surface of the wire rope, the lift between the probe and the rope changes with the scanning position. The amplitude of the differential voltage changes periodically as the probe scans. The distance between the adjacent peaks or valleys is just the space between the adjacent strands and the lay length can be deduced from the scanning signal. The relative error of the lay length obtained by the EC method and the manually measured value is $0.28 \%$.

\section{References}

[1] REN Zhiqian, YU Zongyue, CHEN Xun. Study on wire rope elastic-plastic damage constitutive model[J]. Journal of Mechanical Engineering, 2017, 53(1): 121-129.

[2] ELATA D, ESHKENAZY R, WEISS MP. The mechanical behavior of a wire rope with an independent wire rope core[J]. International Journal of Solids and Structures, 2004, 41(5-6): 11571172 .

[3] VALLAN A, MOLINARI F. A vision-based technique for lay length measurement of metallic wire ropes[J]. IEEE Transactions on Instrumentation \& Measurement, 2009, 58(5): 1756-1762.

[4] WACKER ES, DENZLER J. An analysis-by-synthesis approach to rope condition monitoring[C] //International Symposium on Visual Computing. Springer, Berlin, Heidelberg, 2010: 459-468.

[5] OUELLETTE S. Measurement of lay length of wire rope: USA, US9470657B2[P]. 2016-10-18.

[6] CAO Qingsong, LIU Dan, HE Yuehai, et al. Nondestructive and quantitative evaluation of wire rope based on radial basis function neural network using eddy current[J]. NDT \& E International, 2012, 46: $7-13$.

[7] ZHENG Xinhong, ZHANG Jiayi, ZHANG Xiaolei, et al. An on-line testing device for wire rope lay length: China, CN201520535589.5[P]. 2015-11-18.

[8] FU Fengli, TANG Xiaogao. Asynchronous motor design manual[M]. Beijing: Machinery Industry Press, 2003. 